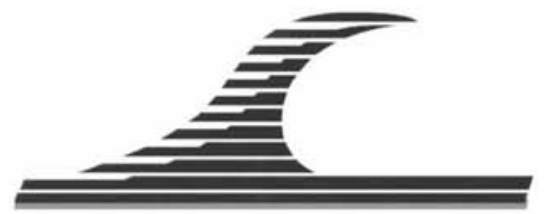

Revue Paralia, Volume 7 (2014) pp s03.1-s03.12

Mots-clés : Télédétection; Cartographie; Conchyliculture; Etat

du domaine; Gestion.

(C) Editions Paralia CFL

\title{
Cartographie de l'état de parcs ostréicoles par télédétection très haute résolution. Application au domaine concédé du Bassin d'Arcachon
}

\author{
Virginie LAFON $^{1}$, Julie MUGICA ${ }^{2}$, Cyril MALLET ${ }^{2}$, Aurélie DEHOUCK ${ }^{1}$ \\ 1. GEO-Transfert, UMR 5805 EPOC, Avenue de facultés, 33405 Talence, France. \\ v.lafon@epoc.u-bordeaux1.fr; a.dehouck@epoc.u-bordeaux1.fr
}

2. BRGM Aquitaine, Parc Technologique Europarc, 24, avenue Léonard de Vinci, 33600 Pessac, France.

j.mugica@brgm.fr; c.mallet@brgm.fr

\section{Résumé :}

Un outil d'aide à la gestion environnementale des parcs ostréicoles du Bassin d'Arcachon est présenté ici. Il repose sur une méthode de détermination semiautomatisée de la nature et de l'état des concessions, exploitant des images de télédétection très haute résolution spatiale. La validation de la carte de télédétection montre que l'état de la surface (entretenu ou en friche) et le mode de culture sont détectés dans $92 \%$ et $85 \%$ des cas, respectivement. D’autre part, les superficies de friche obtenues par télédétection excèdent de $20 \%$ celles mesurées sur le terrain, $75 \%$ des friches étant retrouvées in situ. Au total, 96\% des parcelles sont correctement cartographiées. La télédétection très haute résolution est donc, au vu de ces résultats chiffrés, un outil de gestion du domaine pertinent et efficace, qui, en outre, permet d'enrichir le cadastre d'attributs d'état et de couches vectorisées représentant les friches ostréicoles.

Article issu d'une sélection des XIèmes Journées Nationales Génie Côtier Génie Civil qui se sont tenues aux Sables d'Olonne du 22 au 25 juin 2010. Accepté le 8 mai 2011, en ligne le 27 janvier 2014.

Pour citer cet article :

LAFON V., MUGICA J., MALLET C., DEHOUCK A. (2014). Cartographie de l'état de parcs ostréicoles par télédétection très haute résolution. Application au domaine concédé du Bassin d’Arcachon. Revue Paralia, Vol. 7, pp s03.1-s03.12.

DOI: http://dx.doi.org/10.5150/revue-paralia.2014.s03 
Abstract: An environmental management tool used for mapping oyster-farming activities of the Arcachon lagoon is presented here. It is based on a semi-automatized detection method of the nature and status (clean/dirty) of oyster farms. It exploits very high resolution remote sensing imagery. Validation of the remotely sensed map shows that the surface status and the type of practice are detected in $92 \%$ and $85 \%$ of the cases, respectively. The simulated surface associated to waste lands exceeds by about $20 \%$ the observed ground-field one, $75 \%$ of them corresponding to real "dirty" farms. Altogether, 96\% of the oyster-farming parcels are correctly mapped. Therefore, very high resolution remote sensing is an efficient and valuable management tool that, moreover, allows enhancing the cadaster with attributes relative to the status of oyster farms and underlying waste lands as vector layers.

Keywords: Remote sensing; Mapping; Oyster-farming; Domain status; Management.

\section{Introduction}

Ces travaux se situent dans un double contexte. Ils ont tout d'abord été initiés par le BRGM et GEO-Transfert, la cellule de transfert de technologie du laboratoire EPOC de l’Université Bordeaux 1, afin d'évaluer la faisabilité d'une approche quantitative de qualification par télédétection de l'état du Domaine Public Maritime du Bassin d'Arcachon (LAFON et al., 2010). Ils se poursuivent actuellement dans le cadre de l'inventaire biologique fixé par le Programme Natura 2000 en Mer (financé par l'Agence des Aires Marines Protégées), piloté pour ce site par CREOCEAN en partenariat avec GEO-Transfert pour la mise à disposition exhaustive d'une carte des friches ostréicoles. Ces friches couvrent indifféremment les domaines concédés et non concédés (LAFON, 2010a) du Domaine Public Maritime (DPM). Nous nous focaliserons ici sur la détection de l'état du domaine concédé.

Quoique encore peu développée dans cet objectif, la télédétection est un outil adapté au suivi des zones de production aquacole. Sur les données radar (bande L et X) les exploitations ont une signature brillante liée à la rugosité des coquilles (LEE et al., 2006, DEHOUCK et al., 2011, GADE et al., 2011). Sur les images optiques d'une résolution adaptée aux modes de culture (ex. $50 \mathrm{~cm}$ pour les tables ostréicoles), les cultures de bivalves ont une signature très particulière par rapport à leur environnement liée à la fois à la forme des exploitations, qui constituent des alignements, et à la texture des amas de coquilles (ROUPIOZ et al., 2009). Cette dernière caractéristique a été tout particulièrement exploitée dans le développement d'une chaîne d'analyse d'image orientée vers la caractérisation de l'état des concessions ostréicoles du DPM du Bassin d'Arcachon. Un jeu de vues aériennes rectifiées géométriquement à très haute résolution spatiale a été exploité dans un triple objectif :

- extraire les contours des objets repérables, distinguer les zones productives des friches ostréicoles (infrastructures abandonnées, gisements sauvages, ...), proposer une carte des zones pour lesquelles des travaux de réhabilitation sont nécessaires ; 
- valider l'analyse cartographique faite par télédétection à l'aide d'observations réalisées avec un GPS différentiel et/ou non différentiel ;

- proposer une stratégie robuste pour le suivi de l'état du DPM.

Cette approche a également été testée sur des images satellites afin de proposer un outil d'aide à la gestion moins coûteux.

\section{Méthode}

La méthode d'analyse d'image est détaillée sur la figure 1. Il s'agit d'une chaîne de traitement composée pour discriminer des zones de texture différente et extraire le contour de régions dont la texture révèle des amas d'huîtres cultivées ou non. C’est ici la notion de contraste qui est développée. Plusieurs étapes ont été définies dont la première utilise deux paramètres de texture extraits d'une matrice de co-occurrence (HARALICK et al., 1973) : la moyenne et la variance. L'image de variance est soustraite de l'image afin de réduire une partie du bruit de l'image moyenne. Puis, l'image résultante est lissée à l'aide du filtre de Lee amélioré (LEE, 1980). Généralement appliqué aux images radar pour en réduire le chatoiement, le filtre adaptatif de Lee minimise le bruit aditif aléatoire. Il présente quatre qualités importantes pour nous : celles de minimiser la perte d'information radiométrique et texturale et de préserver les contours tout en réduisant le bruit. Dans notre cas, par rapport à d'autres filtres, il améliore le contraste au niveau des bordures des amas d'huîtres. L'image filtrée est ensuite segmentée en régions de pixels connexes classés en fonction de leur intensité par rapport à un seuil défini par l’opérateur. Cette dernière opération produit une image binaire où les zones productives et amas de coquilles apparaissent en noir sur un fond blanc. Les contours de ces objets sont extraits à l'aide d'un opérateur qui constitue des vecteurs à partir de l'image binaire. Ces contours sont finalement superposés au cadastre ostréicole afin de définir à l'intérieur de chaque parcelle :

- un indicateur d'exploitation (non exploité, culture sur table, culture à plat) ;

- un indicateur d'état (pourcentage de surface hétérogène dans la parcelle).

Ces deux indicateurs sont attribués par l'analyste-expert pour l'aide à la décision. Une reconnaissance de terrain a été réalisée sur quatre sites distincts du DPM du Bassin d'Arcachon afin de valider l'analyse faite à partir des données de télédétection.

L’indicateur d'exploitation et l'indicateur d'état sont validés à l'aide d'observations de terrain. Nous présentons ici les résultats de la validation en nous employant à montrer la pertinence de plusieurs paramètres utiles à la gestion du domaine :

- qualité de la détection des friches ;

- qualité de la détection du mode de culture des parcelles ;

- qualité de la carte des parcelles et de sa table attributaire déterminées par télédétection. 


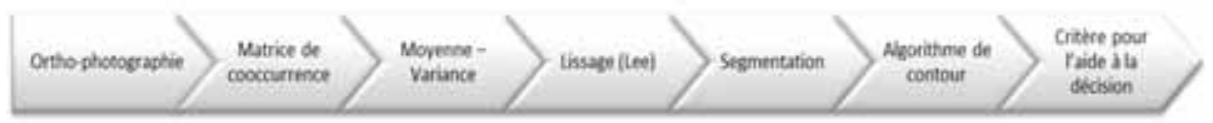

Figure 1. Organigramme du traitement des données images.

\section{Données}

\subsection{Images}

Pour cette étude, nous avons traité des extraits des prises de vue verticales IGN acquises le 27 août 2007 (Observatoire de la Côte Aquitaine), géoréférencées et projetées dans le système Lambert II Etendu. La taille des pixels sur ces photographies est de $50 \mathrm{~cm}$. Ces images géoréférencées seront appelées ortho-photographies dans la suite du document. En complément, nous avons également exploité une image WorldView-1 (WV01) panchromatique d'une résolution spatiale de $50 \mathrm{~cm}$ acquise le 26 avril 2008 à 10:56:39 TU. Elle a été livrée par la société Spot Image en projection UTM Zone 30 (Datum WGS 84). Les traitements sont réalisés sous ENVI.

\subsection{Observations de terrain}

Trois missions d'observation sur le terrain ont permis de contrôler nos résultats. La localisation de ces missions est reportée sur la figure 2.

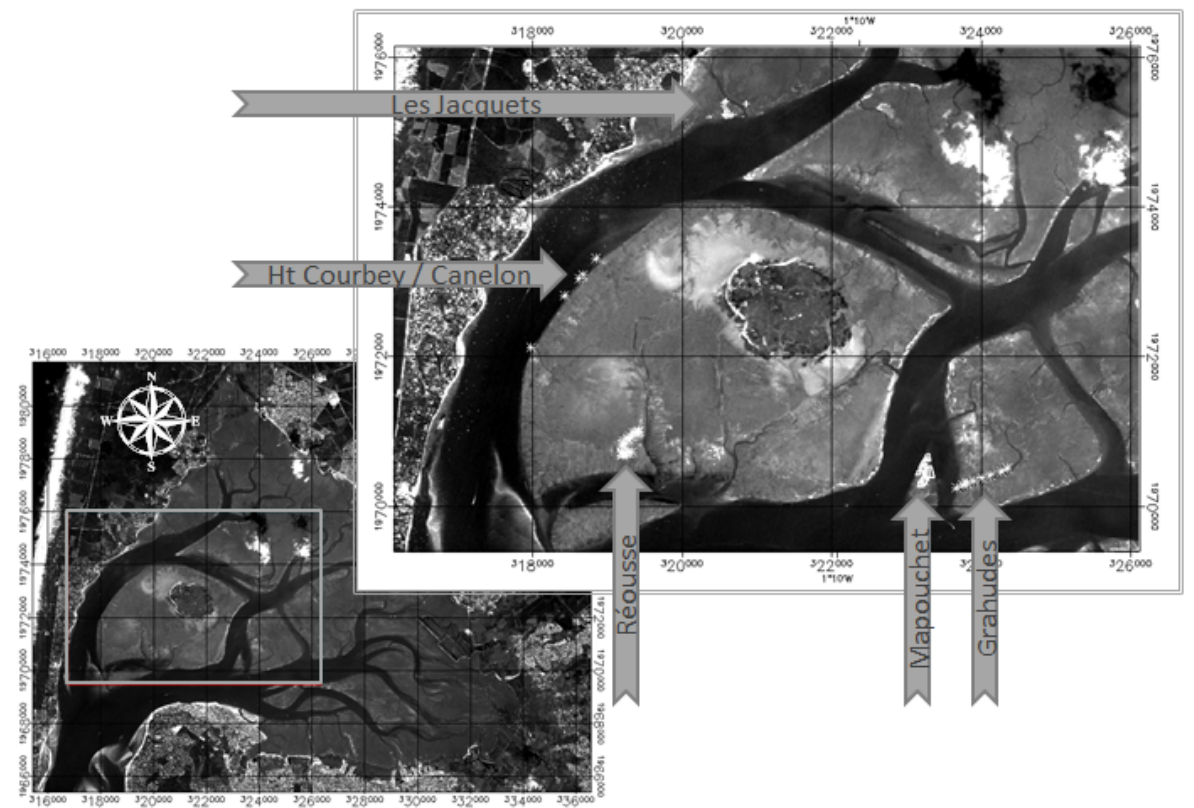

Figure 2. Localisation des données de validation (observations GPS ou DGPS).

La première, exécutée par le service "Cultures Marines et Environnement" de la Direction Départementale des Affaires Maritimes, a été réalisée le 31 mars 2009. Cette 
mission concernait l'étude de l'état du banc de Mapouchet. Les surfaces qui doivent faire l'objet d'un nettoyage ont été identifiées et repérées, puis cartographiées. Ces données seront utilisées pour valider la détection des surfaces en friche ainsi que la cartographie de l'état des parcelles.

La seconde a été exécutée par les Affaires Maritimes, le BRGM et GEO-Transfert le 8 juin 2009. A cette occasion, trois zones ont été visitées : le secteur de la Réousse, un estey du banc des Grahudes et une portion du secteur Haut Courbey / Canelon. Au total, 44 observations DGPS ont été collectées sur ces trois sites. La présence, le type de culture ainsi que l'état de la surface ont été systématiquement renseignés. Ces données permettront de valider la détection des friches dans les parcelles et du mode de culture lorsque celles-ci sont exploitées et en bon état.

La troisième enfin, en date du 26 novembre 2010, a été réalisée sur les concessions des Jacquets (LAFON, 2010b). Au total, ce sont cette fois 19 observations localisées par GPS qui ont été réalisées. A l'instar de la mission de juin 2009, ces données géolocalisées permettront de chiffrer la qualité de détection des friches et du mode de culture à l'intérieur des parcelles ostréicoles.

\subsection{Cadastre ostréicole}

Le cadastre ostréicole a été mis à disposition par le service "Cultures Marines et Environnement" de la Direction Départementale des Affaires Maritimes.

\section{Résultats}

\subsection{Cartographie du site de la Réousse}

Ce site est présenté à part car il a permis de tester à la fois les données satellites et aériennes. En effet, sur les autres sites, l'image WV01 montre des niveaux d'eau un peu élevés pour être exploitable. Le résultat du traitement de l'ortho-photographie et des données satellites d'une partie du site de la Réousse sont reportés sur la figure 3. Celleci présente une superposition de l'ortho-photographie avec les contours (en blanc) extraits automatiquement du traitement de l'image. Les polygones noirs figurent le cadastre ostréicole. Les contours (couche vectorielle), délimitent clairement les couverts d'huîtres. La forme de ces contours permet de déduire tant l'état de chaque parcelle (cultivé ou en friche) que le type de culture associé. Les commentaires sur l'image sont les analyses faites à partir du traitement rédigées à dire d'expert. Ils sont tous validés par des observations de terrain. 


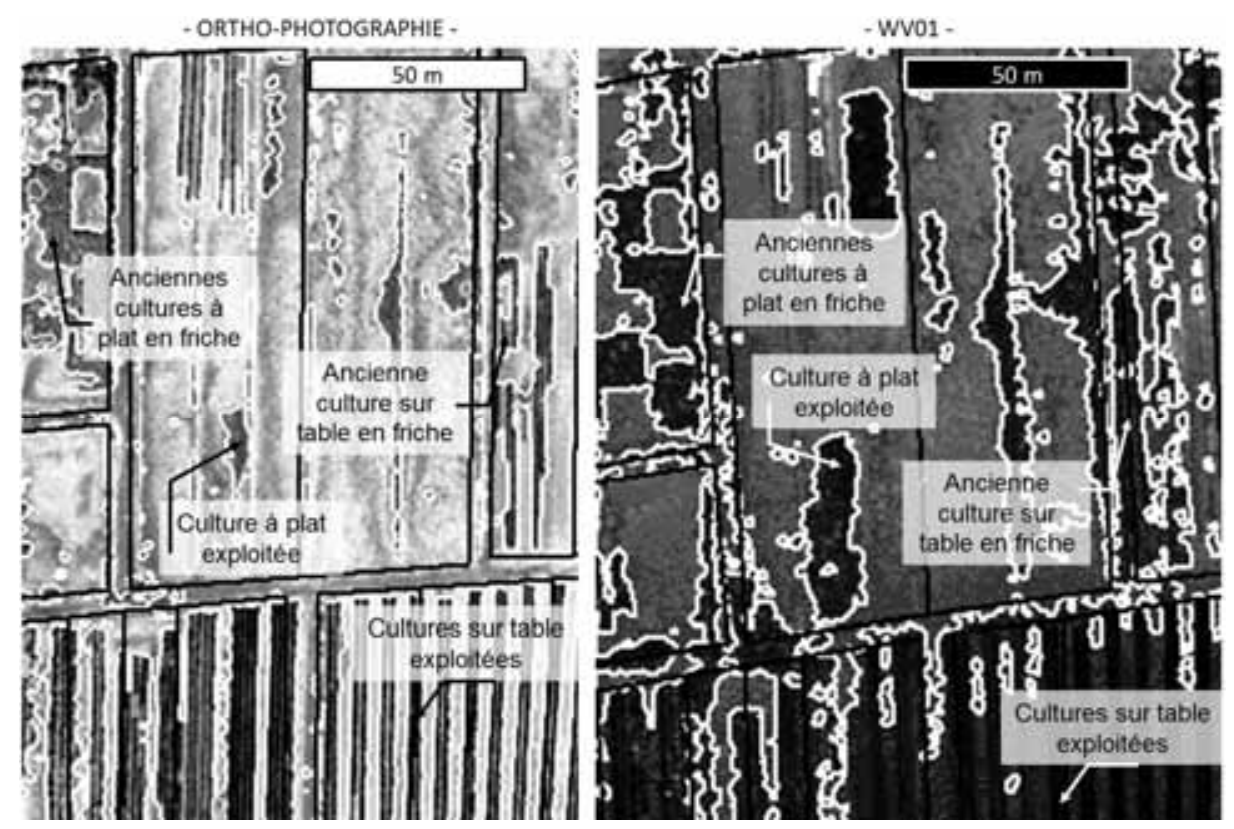

Figure 3. Contours (blancs) extraits du traitement des ortho-photographies (à gauche) et de l'image WV01 (à droite) superposés aux vues originales et au cadastre ostréicole (polygones noirs).

\subsection{Bilan de la détection de l'état du DPM par analyse d'ortho-photographies}

Au total, nous disposons de 63 observations pour valider les cartographies réalisées dont 20 sur le secteur de la Réhousse. Les résultats de la comparaison entre les cartes de télédétection et les observations sont résumés dans le tableau 1.

Tableau 1. Résultats de la validation de la détection de l'état et du mode de culture pour les observations GPS co-localisées aux cartes de télédétection..

\begin{tabular}{llll}
\hline $\begin{array}{l}\text { Observation } \\
\text { Support image }\end{array}$ & \multicolumn{2}{l}{ Secteur Réousse } & $\begin{array}{l}\text { Bilan global } \\
\text { Ortho-photos }\end{array}$ \\
\cline { 2 - 3 } $\begin{array}{l}\text { Indicateur d'état } \\
\text { (friche/propre) }\end{array}$ & $92 \%(58 / 63)$ & WV01 & $95 \%(17 / 20)$ \\
$\begin{array}{l}\text { Indicateur du mode de } \\
\text { culture }\end{array}$ & $81 \%(13 / 16)$ & $31 \%(5 / 16)$ & $85 \%(29 / 34)$ \\
\hline
\end{tabular}

Sur les 20 observations du secteur de la Réhousse, les images WV01 donnent une interprétation correcte de l'état de la surface dans 17 cas sur 20. Les tables sont repérées dans 2 cas sur 8 , les cultures à plat, dans 3 cas sur 8 . L'analyse des ortho-photographies révèle mieux le mode de culture : les cultures à plat sont reconnues dans 5 cas sur 8 et les cultures sur tables sont toujours perçues. De plus, l'état des surfaces est correctement déterminé dans 19 cas sur 20. 
Plus généralement et pour les 63 observations, dans 92\% des cas, les observations GPS corroborent l'indicateur d'état déduit du traitement d'image. Les principaux échecs concernent :

- dans quatre cas des dépôts de coquilles de très petites dimensions qui ne sont pas mis en évidence par la méthodologie proposée. Dans un des cas la friche, dimensionnée sur le terrain, fait environ $3 \mathrm{~m}$ de diamètre, ce qui nous permet de chiffrer un seuil de détection des friches en fonction de leur taille ;

- dans un cas une ancienne culture sur table, en friche, analysée comme une culture sur table en bon état.

La reconnaissance du mode de culture est, globalement, de 85\%. Mais cette valeur n'est pas très représentative, car 26 cultures sur tables contre seulement 8 cultures à plat servent de base à cette analyse. Les cultures sur tables sont reconnues dans 24 cas sur 26 (soit plus de 92\%). Dans les 2 cas non détectés, les tables, sur le terrain (novembre 2010) étaient couvertes de collecteurs (figure 4, zoom I, a).

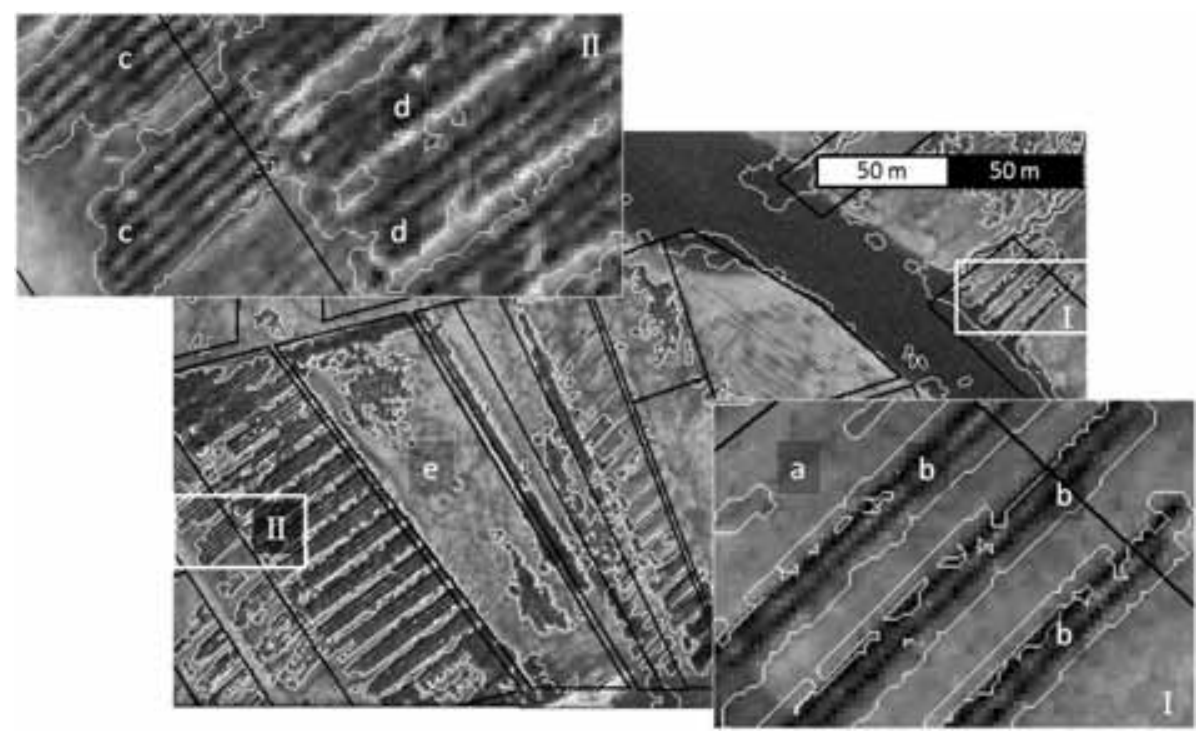

Figure 4. Contours extraits du traitement de l'ortho-photographie des Jacquets (contours blanc) superposés au cadastre ostréicole (polygones noirs). Légendes : a : collecteurs vides ; $b$ : collecteurs pleins ; $c$ : culture sur table entretenue ; $d$ : culture sur table en friche; $e$ : culture à plat en friche.

Seuls les supports des collecteurs apparaissent en filigranes sur l'ortho-photographie. Des collecteurs pleins observés sur le terrain sont correctement cartographiés sur la même image (figure 4, zoom I, b). Les collecteurs offrent une apparence différente des tables couvertes de poches d'huîtres. En effet, les ils se présentent sous forme de fines lignes très sombres, les alignements de poches sont légèrement plus clairs et aussi plus larges (figure 2, figure 4, zoom II, c). Hors mis ces tables de collecteurs, vraisemblablement vides au moment de la prise de vue, l'ensemble des tables exploitées 
est reconnu. Il est également très intéressant de noter que d'anciennes tables, réduites à l'état de friches sont également repérées par traitement des ortho-photographies (figure 4, zoom II, d).

Les cultures à plat sont reconnues dans 5 cas sur 8 (figure 3 et figure 4, e). Ce score est relativement faible du fait de la difficulté à discriminer dans certains cas les cultures à plat en friches de friches ostréicoles sans aucune structuration. Dans un cas une culture à plat en friche est interprétée comme une culture sur table en friche. Cette analyse montre qu'il est nécessaire d'établir un catalogue de référence pour les cultures à plat qui permette une analyse plus fine des images traitées.

\subsection{Bilan de l'analyse cartographique}

Pour cette analyse, nous avons traité l'ortho-photographie du banc de Mapouchet, couvert de 52 parcelles ostréicoles. A partir des vecteurs issus du traitement, la superficie totale des zones en friche (figure 5) s'élève à 3,7 ha, soit $20 \%$ de plus que celle déterminée sur le terrain. La surface révélée douteuse par analyse d’images recoupe 2,1 ha de la surface cartographiée in situ. Au total, 19 concessions présentent des hétérogénéités de surface sur plus de 10\% de leur superficie, 16 d'entre elles correspondent aux observations in situ (figure 5, encadré blanc et noir). Les concessions propres ne sont pas reportées ici pour ne pas surcharger la figure.

Trois concessions sont abusivement déclarées en friche par analyse des orthophotographies (figure 5, encadré noir). Pour la concession localisée au sud-est de la figure, ni les résultats du traitement, ni l'expertise ne peuvent être mis en doute : les exploitations sont couvertes de coquilles. La cartographie de terrain ne les révèle pas. Toutefois, la DDTM nous a informés, au vu de ces clichés, que des travaux de réhabilitation avait été entrepris entre le levé survol et la mission de terrain, ce qui explique qu'elle n'apparaisse plus comme une concession en friche sur leurs propres observations. Cette concession ne peut être considérée dans l'analyse de validation. Pour les concessions encadrées de noir situées au nord et au sud, l'expertise menée à partir du traitement des ortho-photographies est plus discutable.

Au nord, le contour extrait de l'analyse de texture révèle en fait la limite du plan d'eau. Ainsi, le niveau d'eau limite l'applicabilité du traitement et empêche une analyse correcte de la concession. Au sud, plusieurs contours irréguliers apparaissent dans la concession. Toutefois la couleur semble indiquer la présence de végétation et non de coquilles. 


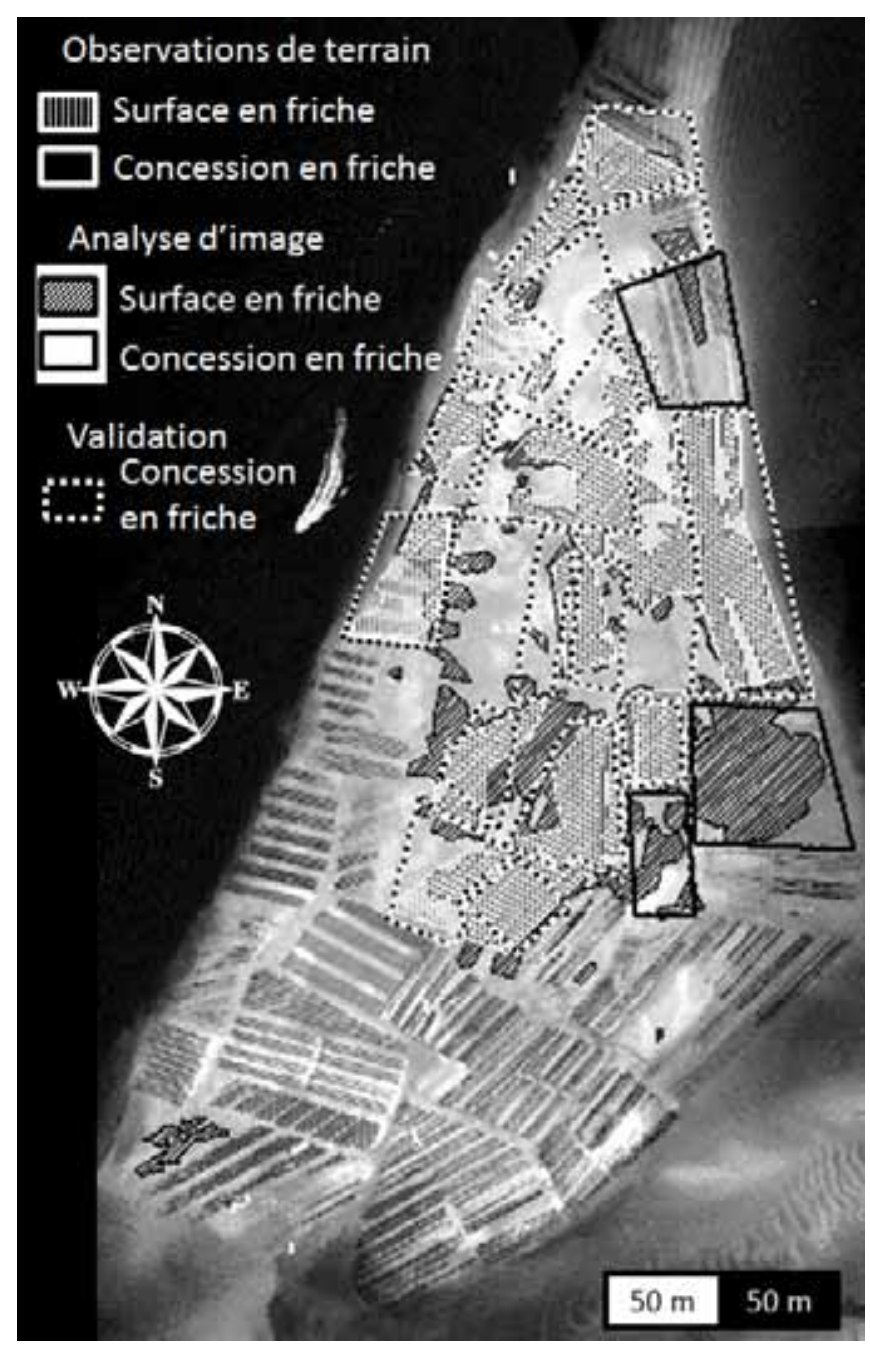

Figure 5. Cartographie des friches ostréicoles sur le banc de Mapouchet.

\section{Discussion des résultats et conclusions}

Ces travaux montrent l'utilité de la télédétection très haute résolution pour la cartographie de l'état du Domaine Public Maritime dans le cas du Bassin d'Arcachon, qui permet une expertise des estrans au cours de la marée basse. Le gestionnaire du domaine doit en constater annuellement l'état d'entretien, ce qui entraîne d'importants frais de mission sur le terrain. Une méthode de traitement d'image adaptée à l'usage d'ortho-photographies d'une résolution de $50 \mathrm{~cm}$ a été développée et validée par des observations GPS et DGPS. Cette méthode, basée sur une analyse de texture, permet de générer le contour des surfaces couvertes de coquilles d'huître. La présence et la forme de ces contours sont une aide précieuse à la décision, car elles permettent de détecter dans de nombreux cas le mode de culture ainsi que l'état d'entretien de celles-ci. Cette détection n'est pas encore automatique.

Ainsi, l'état de surface (entretenu/en friche) est révélé dans plus de 92\% des cas. Une carte a été générée pour un banc couvert d'exploitations et comparée à une carte déduite 
d'observations sur le terrain. Les concessions portent des attributs identiques dans plus de $96 \%$ des cas. L'utilisation des ortho-photographies est limitée par le niveau d'eau lié à la marée Il est nécessaire d’acquérir les prises de vue à marée basse, de préférence en période de fort coefficient. La taille des friches est également un facteur limitant. Les friches d'une taille inférieure à trois mètres de diamètre ne sont pas repérables. Les lissages successifs appliqués expliquent cette perte d'information. Enfin, pour parfaire la qualité des cartes extraites des ortho-photographies traitées, il faudra ajouter à l'analyse de la rugosité une analyse spectrale (qui tienne compte de la couleur) afin, le cas échéant, de ne pas confondre des zones d'herbiers avec un couvert de coquilles. Les zones d'herbiers dans les parcs sont toutefois très peu fréquentes et restent un problème marginal. Il faut également noter que les dépôts d'algues vertes, aux saisons où les proliférations sont marquées peuvent également être une source d'erreur. Là encore, une analyse spectrale peut les révéler efficacement.

Les ortho-photographies permettent également de déterminer dans de nombreux cas la présence d'une structuration des friches. Ainsi, il est possible de détecter des déchets de nature particulière qui peuvent être associés aux friches, les tables en particulier. Cette connaissance permet au gestionnaire d'apprécier plus finement le temps nécessaire, les engins indispensables et finalement les coûts des opérations de nettoyage. Enfin, en ce qui concerne le suivi des zones de culture, la méthode d'analyse de texture permet de mettre en évidence dans $92 \%$ des cas les cultures sur tables. Les cultures à plat sont reconnues moins fréquemment (dans 5 cas sur 8 seulement). Pour lever une partie de la difficulté à définir un indicateur d'exploitation correct, il est nécessaire, pour ce type de culture en particulier, d'établir un catalogue de référence pour l'analyste.

Cette approche permet de renseigner les gestionnaires de deux façons. D’une part, en croisant sous SIG les couches vectorielles issues du traitement avec le cadastre ostréicole, il est possible d'enrichir ce dernier en affectant des attributs d'état et le mode de culture par concession. D’autre part, la génération de polygones permet de déduire les superficies couvertes par les huîtres exploitées et sauvages et donc de participer à la quantification des stocks d'huîtres dans le bassin. Ces informations sont très utiles non seulement pour mieux prioriser et budgétiser les opérations de nettoyage des friches, mais également, dans le contexte de la crise de mortalité des huîtres de valoriser les récifs d’huîtres sauvages.

Afin de réduire le coût des données traitées, la méthode développée a également été appliquée à des données satellites. Celles-ci, malgré leur résolution (50 cm également), semblent un peu moins adaptées que les ortho-photographies, notamment en ce qui concerne la détection du mode de culture. La chaîne de traitement révèle moins efficacement les contrastes, notamment ceux formés par des alignements que dans le cas des ortho-photographies. Pour traiter ce type de données, une approche basée sur des filtres permettant de révéler des éléments texturés dans l'image, typiquement des alignements formés par les tables ostréicoles, est en cours de développement dans le 
cadre d'un stage financé par le programme CNES/ORFEO (programme d'accompagnement au lancement des satellites PLEIADES). Cette méthode doit permettre en un seul traitement de dégager l'ensemble des cultures sur table. Les gisements naturels (hors structuration anisotrope lié à la colonisation d'anciennes tables), mais également les herbiers et les dépôts d'algues, ne seront plus détectés. De plus, cette méthode est vraisemblablement moins sensible au niveau d'eau. Ces travaux permettent enfin d'approcher une méthode plus opérationnelle car impliquant moins largement un expert analyste. Ceci pourrait donc réduire de façon importante les coûts de cartographie. Cette nouvelle approche offre donc de nombreuses perspectives.

\section{Remerciements}

Nous remercions le service "Cultures Marines et Environnement" de la Direction Départementale des Affaires Maritimes de la Gironde, pour leur coopération et leur disponibilité sur le terrain.

\section{Références bibliographiques}

DEHOUCK A., LAFON V., BAGHDADI N., ROUBACHE A., RABAUTE T. (2011). Potential of TerraSAR- $X$ imagery for mapping intertidal coastal wetlands. Proceedings of the $4^{\text {th }}$ TerraSAR-X Science Team Meeting, Oberpfaffenhofen, 8 p.

GADE M., SELTZER K., KHOLUS J. (2011). On the Use of Multi-Frequency SAR Imagery for a Surveillance of the Wadden Sea Ecosystem on the German North Sea Coast. Proceedings of the $4^{\text {th }}$ TerraSAR-X Science Team Meeting, Oberpfaffenhofen, $8 \mathrm{p}$.

HARALICK R., SHANMUGAM K., DINSTEIN I. (1973). Textural features of image classification. IEEE Transactions on. Systems, Man and Cybernetics, Vol. 6, pp 610-621. http://dx.doi.org/10.1109/TSMC.1973.4309314

LAFON V. (2010a). Inventaires biologiques et analyse écologique de l'existant, Natura 2000 en Mer, Lot 3 - Arcachon. Rapport d'Avancement n². GEO-Transfert UMR 5805 EPOC.

LAFON V. (2010b). Inventaires biologiques et analyse ecologique de l'existant, Natura 2000 en Mer, Lot 3 - Arcachon. Rapport de mission $n^{\circ} 1$ - 26/11/2010. Les Jacquets, GEOTransfert UMR 5805 EPOC.

LAFON, V., MUGICA J., MALLET C. (2010). Cartographie de l'état du Domaine Public Maritime par télédétection très haute résolution: Application au Bassin d'Arcachon. XI ${ }^{\text {èmes }}$ Journées Nationales Génie Côtier - Génie Civil, Sables d'Olonne 22-25 Juin, pp 505-512. http://dx.doi.org/10.5150/jngcgc.2010.059-L

LEE J.-S. (1980). Digital Image Enhancement and Noise Filtering by Use of Local Statistics. IEEE Transactions on Pattern Analysis and Machine Intelligence, Vol. PAMI-2, n 2, pp 165-168. http://dx.doi.org/10.1109/TPAMI.1980.4766994 
s03.12 : Revue Paralia - Vol. 7 (2014)

LEE S.-K., HONG S.-H., KIM S.-W.YAMAGUSHI Y., WON, J.-S. (2006). Polarimetric features of oyster farm observed by AIRSAR and JERS-1. IEEE Transactions on Geoscience and Remote Sensing, Vol. 44, $n^{\circ} 10$, pp 2728-2735. http://dx.doi.org/10.1109/TGRS.2006.879107

ROUPIOZ L., SCHMIDT A., KRAMER H., FEY F., CREMER J., DIJKMAN E., JANSEN J., DANKER N. (2009). Use of Remote Sensing methods in monitoring mussel beds in the Wadden Sea. $4^{\text {th }}$ EARSeL Workshop on Remote Sensing of the Coastal Zone. 